\title{
CARACTERIZACIÓN DE DIATOMITA POR DIFRACCIÓN DE RAYOS X
}

\author{
(Characterization of Diatomite \\ by X-Ray Diffraction)
}

Carlos E. Vargas Infante, Gabriela Jiménez Sánchez, Wilson E. Naranjo M.

\begin{abstract}
Escuela de Ingeniería Geológica, Uptc-Sogamoso, Grupo de Investigación Ingeniería Geológica, cevi600@gmail.com - gabyjimsan@hotmail.com
\end{abstract}

(Recibido Septiembre 8 de 2006 y aceptado Abril 18 de 2007)

\section{Resumen:}

La técnica de difracción de rayos $\mathrm{X}$ se utilizó para hacer una caracterización detallada de los minerales arcillosos presentes en la diatomita de un yacimiento que no había sido objeto de estudio ni exploración que permitiera determinar su potencial tanto minero como industrial, específicamente en el área piloto del sector Tunja-Chivatá (Boyacá).

Palabras clave: Diatomita, Beneficio, Caracterización.

\section{Abstract:}

An X-Ray diffraction technique was used to a detail characterization of the clay minerals in a diatomite deposit, which has not been studied nor explored, in order to determine its mining and industrial potential, located in the Tunja-Chivatá (Boyacá) pilot area.

\section{INTRODUCCIÓN}

$\mathbf{L}$ a diatomita tiene gran importancia en la industria, como filtro, como material refractario, en fertilizantes, catalizadores químicos, pesticidas y otros. Este estudio se basa en la aplicación de la técnica de difracción de rayos $\mathrm{X}$ a muestras obtenidas de un yacimiento de diatomita que no había sido objeto de estudio ni exploración que permitiera determinar su potencial tanto minero como industrial, específicamente en el área piloto del sector Tunja-Chivatá.

Para la caracterización general del yacimiento se tiene una primera fase que corresponde a la caracterización petrográfica, como parte de la caracterización mineralógica, y de cuyos resultados se identifican diatomeas y minerales como cuarzo, feldespatos, minerales arcillosos, agregados de óxidos e hidróxidos de $\mathrm{Fe}$ (goethita). De tal forma, y siguiendo la metodología planteada, se realizan ensayos de difracción de rayos $\mathrm{X}$.

Sabiendo de antemano el grado de complejidad que tiene la diferenciación de minerales arcillosos a nivel petrográfico, la técnica de difracción de rayos $\mathrm{X}$ permite de manera detallada hacer la caracterización de las distintas fases de los minerales, tales como la illita, esmectita y caolinita, como también determinar el grado de cristalización de ellos basados en el difractograma por las reflexiones estrechas y simétricas, lo cual Facilita el análisis de resultados de muestras producto del proceso de beneficio.

\section{MÉTODO EXPERIMENTAL}

Siguiendo el procedimiento de preparación para muestras orientadas, todas las partículas de los minerales arcillosos deben asentarse por sedimentación (agregados orientados) o
Ser dispuestas paralelamente por espaciamiento (pasta orientada), así sus ejes A y B o sus planos ab quedarán paralelos al portaobjetos y todos los planos orientados paralelamente difractarán en el mismo momento.

Para este procedimiento se toma una muestra de aproximadamente $50 \mathrm{~g}$ y se pulveriza. De esta forma se toman $10 \mathrm{~g}$ de la muestra para mezclarla y diluirla en $100 \mathrm{ml}$ de agua destilada para luego ser agitada durante 15 minutos; después de agitada la muestra se coloca en una probeta de $100 \mathrm{ml}$ por 50 minutos para que se decante y así garantizar que la fracción fina y orientada quede en la parte superior de la probeta; $y$ con la pipeta se procede a extraer una muestra de esta introduciéndola $2 \mathrm{~cm}$ aprox., para luego colocarla sobre los portaobjetos (IGAC, 1995).

Se elaboran tres montajes para cada punto de muestreo, para realizar las pruebas de difracción de rayos $\mathrm{X}$ correspondientes a barrido en natural, etilenglicol y calentada a $550{ }^{\circ} \mathrm{C}$ (Thorez, 2003).

\section{RESULTADOS}

\subsection{Análisis petrográficos}

A partir del análisis petrográfico (análisis previo a la difracción) se obtuvieron los minerales ópalo (constituyente de la frustura de la diatomea), minerales arcillosos, turmalina, zircón, cuarzo, agregados de óxidos e hidróxidos de $\mathrm{Fe}$ (goethita) y feldespatos.

\subsection{Análisis de difracción de rayos $X$}

En el difractograma (ver figura 1) de la muestra en polvo (fracción total) DA1-T se determina la presencia de los minerales: 
esmectita, illitas, caolinita, cuarzo, biotita y moscovita. E1 análisis se basa en los datos de las reflexiones, los cuales coinciden en varios casos con dos minerales a la vez, lo que dificulta la diferenciación de minerales como la illitas y las micas, debido a que se encuentran como mezcla, pero facilita la caracterización general del yacimiento, en este caso el reconocimiento del amorfo estudiado en este artículo, correspondiente al ópalo y micas (biotitas y moscovita). Este método hace una descripción más cualitativa que cuantitativa.

A partir de los difractogramas (ver figura 2) de las muestras orientadas se realiza una semicuantificación de los minerales presentes con base en valores de intensidad (cps) y las reflexiones basales de los minerales dominantes.
A partir de los datos obtenidos en las tablas 1 y 2 se evidencia una variación porcentual de los minerales caolinita y el cuarzo, mientras se mantiene constante en la illita y con relativa varianza en la esmectita de la muestra DA1-T, correspondiente al afloramiento respecto a la muestra DP1-8 de la base, lo cual indica que los estudios de beneficio se deben intensificar en la parte superior del yacimiento.

Para determinar el grado de cristalización se tiene en cuenta la distancia interplanar entre los ángulos superiores e inferiores respecto al eje de simetría correspondiente a los triángulos dados por la reflexión que determina cada uno de los minerales (ver tabla 3).

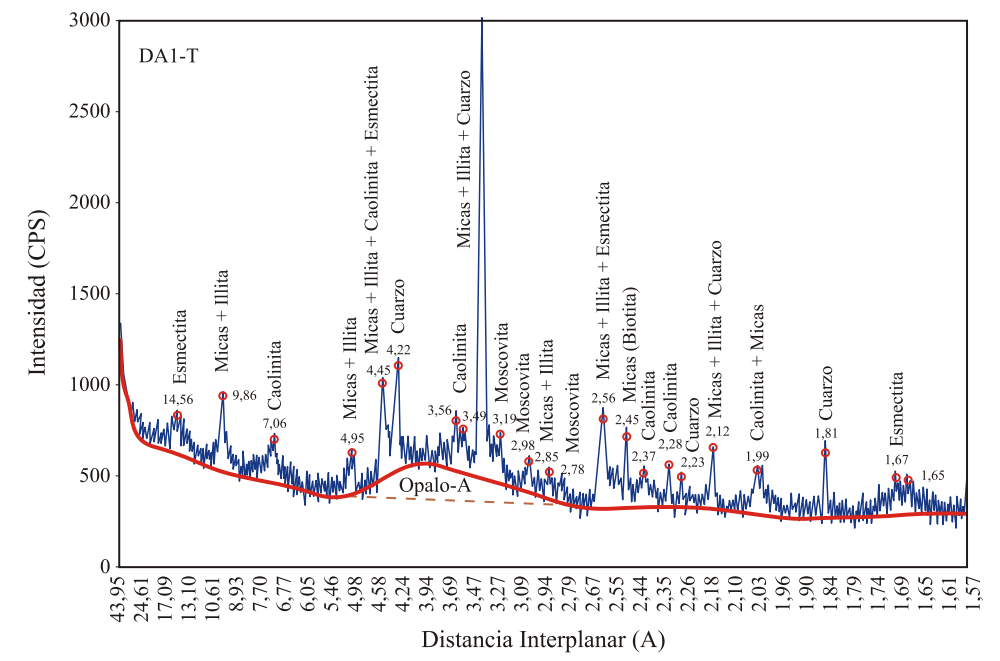

Figura 1. Difractograma de muestra en polvo de la muestra DA1-T.

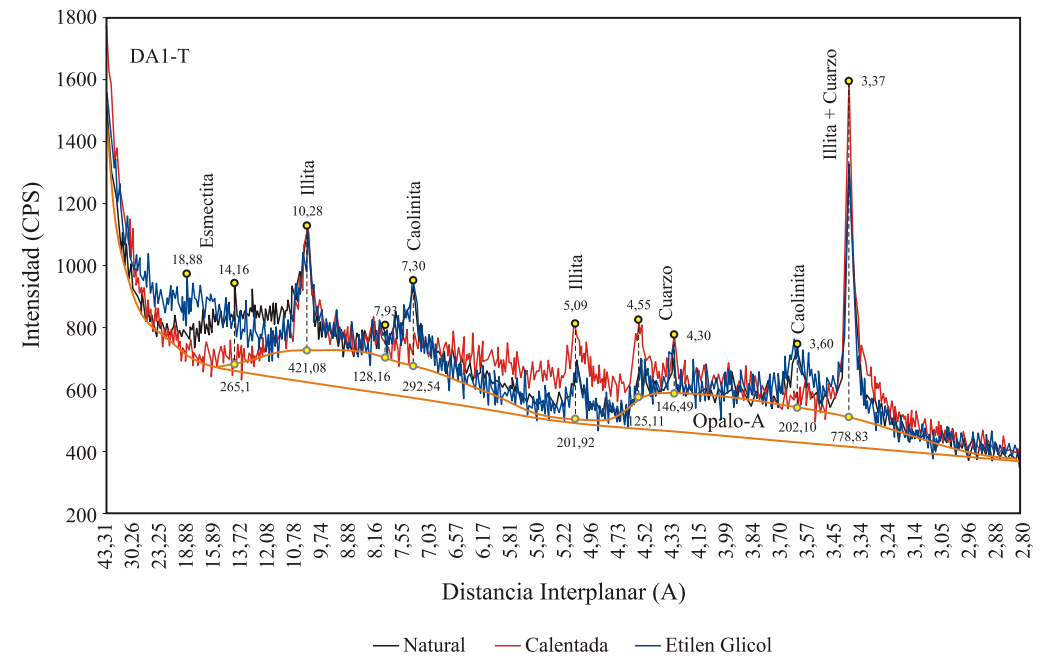

Figura 2. Identificación de minerales y mineraloides en la muestra DA1-T. 
Tabla 1. Cuantificación mineralógica por difracción de rayos $X$, fracción $<2 \mu$ para da1-t.

\begin{tabular}{|c|c|c|c|c|c|c|}
\hline Minerales & Composición química & $\begin{array}{c}\text { Reflexión } \\
\text { basal }\end{array}$ & $\begin{array}{c}\text { Intensidad } \\
(\%)\end{array}$ & $\begin{array}{c}\text { cps } \\
\text { Recálculo } \\
\text { deps }\end{array}$ \\
\hline Illita & $\mathrm{KAl}_{2} \mathrm{Si}_{2} \mathrm{O}_{5}(\mathrm{OH})_{4}$ & 10,28 & 100 & 421,08 & 421,08 & 30,14 \\
\hline Caolinita & $\mathrm{Al}_{2} \mathrm{Si}_{2} \mathrm{O}_{5}(\mathrm{OH})_{4}$ & 7,3 & 100 & 292,54 & 292,54 & 20,94 \\
\hline Esmectita & $(\mathrm{Ca}, \mathrm{Na})\left(\mathrm{Si}_{4 \mathrm{x}} \mathrm{Al}_{\mathrm{x}}\right)(\mathrm{Al}, \mathrm{Fe}, \mathrm{Mg})_{3} \mathrm{O}_{20}(\mathrm{OH})_{2}$ & 14,16 & 100 & 265,1 & 265,1 & 18,97 \\
\hline Cuarzo & $\mathrm{SiO}_{2}$ & 4,3 & 35 & 146,49 & 418,54 & 29,95 \\
\hline & & & & $\sum=1397,26$ & $\sum=100$ \\
\hline
\end{tabular}

Tabla 2. Cuantificación mineralógica por difracción de rayos X, Fracción $<2 \mu$ para DP1-8.

\begin{tabular}{|c|c|c|c|c|c|c|}
\hline Minerales & Composición química & $\begin{array}{c}\text { Reflexión } \\
\text { basal }\end{array}$ & $\begin{array}{c}\text { Intensidad } \\
(\%)\end{array}$ & $\begin{array}{c}\text { cps } \\
\text { Recálculo } \\
\text { cps }\end{array}$ \\
\hline Illita & $\mathrm{KAl}_{2} \mathrm{Si}_{2} \mathrm{O}_{5}(\mathrm{OH})_{4}$ & 10,40 & 100 & 1271,1 & 1271,1 & 30,01 \\
\hline Caolinita & $\mathrm{Al}_{2} \mathrm{Si}_{2} \mathrm{O}_{5}(\mathrm{OH})_{4}$ & 7,4 & 100 & 1356,26 & 1356,26 & 32,02 \\
\hline Esmectita & $(\mathrm{Ca}, \mathrm{Na})\left(\mathrm{Si}_{4 \mathrm{x}} \mathrm{Al}_{\mathrm{x}}\right)(\mathrm{Al}, \mathrm{Fe}, \mathrm{Mg})_{3} \mathrm{O}_{20}(\mathrm{OH})_{2}$ & 15,95 & 100 & 932,38 & 932,38 & 22,01 \\
\hline Cuarzo & $\mathrm{SiO}_{2}$ & 4,3 & 35 & 236,66 & 676,17 & 15,96 \\
\hline & & & & $\sum=4235,91$ & $\Sigma=100$ \\
\hline
\end{tabular}

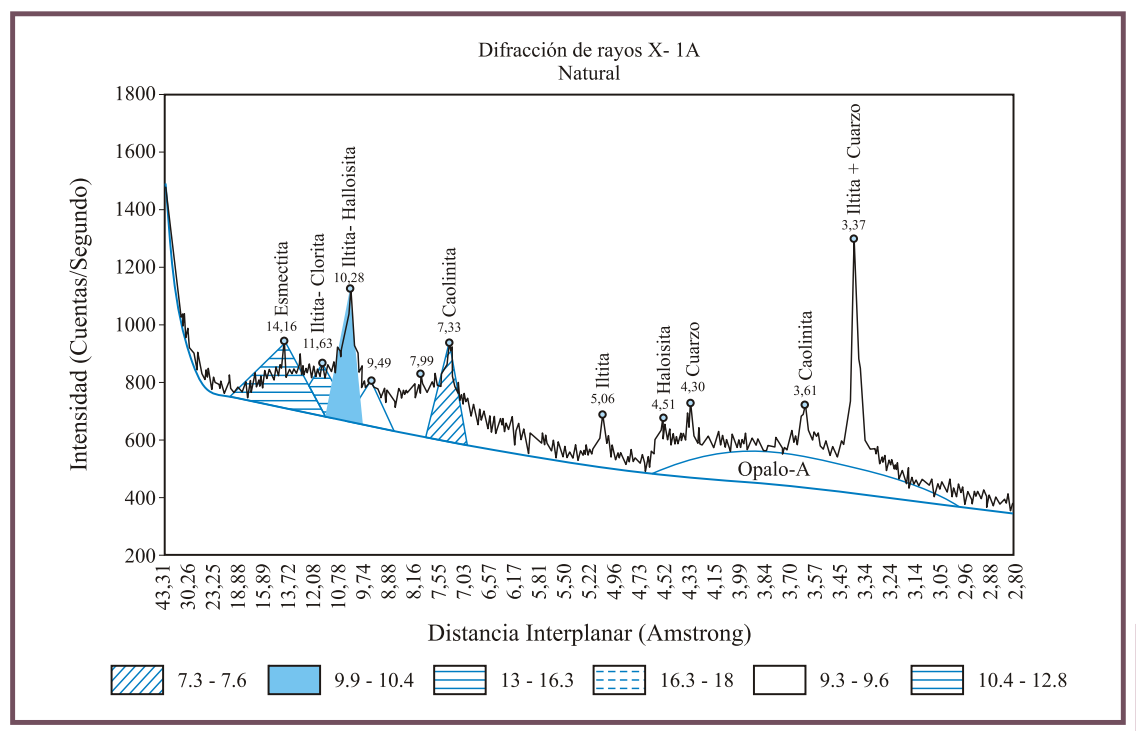

Figura 3. Determinación del grado de cristalización DA1-T.

Tabla 3. Relación de simetría de illita, caolinita, esmectita y cuarzo

\begin{tabular}{|c|c|c|c|c|c|c|c|c|c|}
\hline \multirow[b]{2}{*}{ Muestra } & \multicolumn{3}{|c|}{ 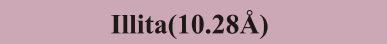 } & \multicolumn{3}{|c|}{ 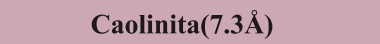 } & \multicolumn{3}{|c|}{ Esmectita(14.5Å) } \\
\hline & $\begin{array}{l}\text { Dist. } \\
\text { Ángulos } \\
\text { bajos }\end{array}$ & $\begin{array}{l}\text { Dist. } \\
\text { ángulos } \\
\text { altos }\end{array}$ & $\begin{array}{c}\text { Índice } \\
\text { de } \\
\text { simetría }\end{array}$ & $\begin{array}{l}\text { Dist. } \\
\text { ángulos } \\
\text { bajos }\end{array}$ & $\begin{array}{l}\text { Dist. } \\
\text { ángulos } \\
\text { altos }\end{array}$ & $\begin{array}{c}\text { Índice } \\
\text { de } \\
\text { simetría }\end{array}$ & $\begin{array}{l}\text { Dist. } \\
\text { ángulos } \\
\text { bajos }\end{array}$ & $\begin{array}{l}\text { Dist. } \\
\text { ángulos } \\
\text { altos }\end{array}$ & $\begin{array}{c}\text { Índice } \\
\text { de } \\
\text { simetría }\end{array}$ \\
\hline DA1-T & 10,4 & 9,9 & 1,05 & 7,6 & 7,3 & 1,04 & 12,8 & 10,4 & 1,23 \\
\hline DP1-8 & 7,6 & 7,3 & 1,04 & 10,4 & 9,9 & 1,05 & 16,3 & 13 & 1,25 \\
\hline
\end{tabular}


De acuerdo con la figura 3, el grado de cristalización está dado de la siguiente manera: de menor a mayor grado, esmectita, illita, y caolinita; la esmectita es el mineral que más desordenado se encuentra, por lo tanto, muestra una diferencia notable respecto a sus ángulos superiores e inferiores. Luego se encuentra la illita y sucesivamente la caolinita, dado por la distancia entre los ángulos superiores e inferiores respecto al eje de simetría. Mientras que en la figura 4, de menor a mayor grado de cristalización, están la esmectita, la caolinita y la illita.

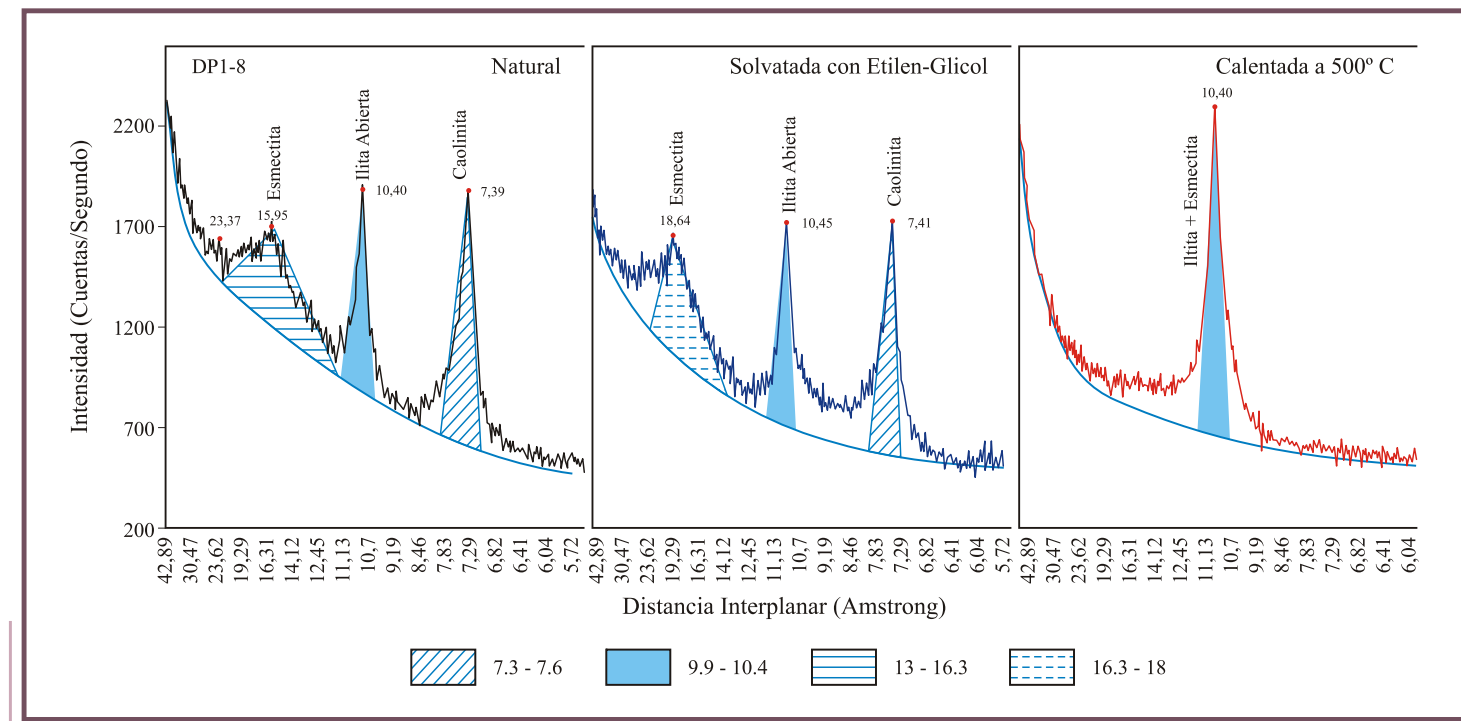

Figura 4. Determinación del grado

de cristalización DP1-8.

\section{CONCLUSIONES}

Para los estudios de minerales de uso industrial, como la diatomita, es importante determinar el grado de cristalización de los minerales constituyentes, partiendo de los difractogramas, las distancias de los ángulos y el cálculo del índice de simetría.

A partir del análisis de resultados de la tabla 3 es notable una variación en el grado de cristalinidad de la illita en la muestra correspondiente a la base DP1-8, con relación a la de la muestra de afloramiento DA1-T.

En los tres tipos de montajes, el natural, etilenglicol y calentada a $550{ }^{\circ} \mathrm{C}$, se puede apreciar las variaciones que pueden llegar a sufrir los minerales al ser atacados por los químicos y los cambios de temperatura, y de esta manera correlacionar estos datos con otras caracterizaciones de muestras naturales con el beneficio.

La difracción de rayos $\mathrm{X}$ es el método experimental más exacto a la hora de identificar, cuantificar y clasificar minerales arcillosos.
La muestra DA1-T presenta una variación en porcentaje de sus minerales constituyentes como son el cuarzo y la caolinita, mientras que permanece estable la illita, y la esmectita no presenta mayor variación.

Los rangos obtenidos en los difractogramas, para las muestras en polvo orientadas y de fracción total, corresponden a los valores normales de los mismos obtenidos por tablas.

A partir de los análisis de DRX se puede concluir que el área de estudio es viable para explotación en su fase preliminar, y se recomienda la realización de más pruebas para garantizar su rendimiento en el uso industrial.

\section{REFERENCIAS}

IGAC (1995). Suelos de Colombia -Caracterización de los productos de alteración; arenas y arcillas en los suelos de Colombia. Bogotá: Ed. Canal Ramírez.

Thorez, J. (2003). Determination of Clay Minerals by X-Ray Difraction and Application in Clay Geology. Universite de Liege. Bélgica. 